\title{
Digital body maps reinvent the way we perceive and discuss pain and discomfort
}

\author{
DOI: https://doi.org/10.5114/pq.2018.79739
}

\section{Shellie A. Boudreau}

Department of Health Science and Technology, Aalborg University, Aalborg, Denmark

\begin{abstract}
The revival of pain drawings as digital body maps for visualizing pain and discomfort changes the way we understand patients and communicate with them. Data stemming from simple drawings of musculoskeletal pain helped reveal the significance and impact of single versus multi-site pain reports. To achieve the next big step, we will need to go beyond pain site assessment and capture a more complete clinical picture of pain as it unfolds. Recent digital pain mapping studies show hidden patterns of pain expression within these marked pain sites. This mini-review reflects on the knowledge obtained from simple paper pain drawings, touches upon new digital technologies, and discusses how these technologies will allow us to reinvent the way we perceive and discuss pain.

Key words: e-health, pain drawing, digital pain drawing, pain map, pain sites, pain location
\end{abstract}

\section{Introduction}

The revival of pain drawings as digital body maps for visualizing pain [1-5] and discomfort changes the way we understand patients and communicate with them [1]. A review of the literature following the introduction of pain drawings suggests that little information about a patient's condition can be extracted from the pain drawing alone. For many years, pain drawings were performed on simple body charts reflecting an androgynous outline of the human body or body parts, with little or no anatomical detail, as shown in Figure 1. Furthermore, the instructions on how to perform a pain drawing differed across studies. As an example, patients indicated the general location of their pain by marking an $X$, selecting

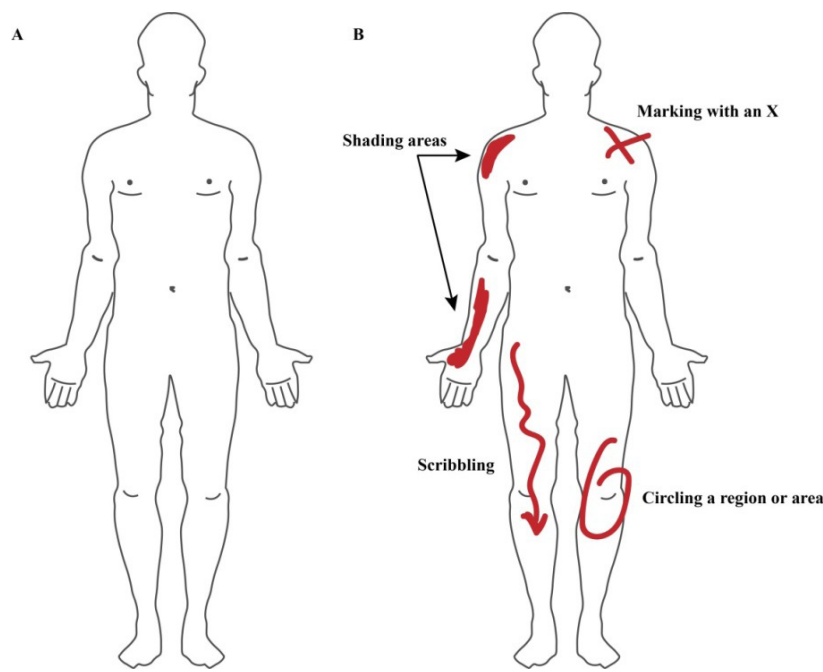

Figure 1. (A) A traditional and simple outline of an androgynous body, often used in paper-based drawings, and (B) common reporting characteristics for completing pain drawings, e.g. marking with an $\mathrm{X}$, circling a region, shading an area, or scribbling a body region, drawing a circle, or shading an area. Sometimes scribbles or arrows were used to specify complex expressions or possibly radiating pain. Without a doubt, the integrity of these earlier drawings and simplicity of the body charts could create debatable interpretations. For that reason, researchers reduced a patient's expression of their pain to results based on generalized location.

Fortunately, a number of research groups continued to acquire pain drawings and pain location information despite what may have been viewed as a limitation. Through incremental steps, researchers began to uncover important associations between the locations of pain and symptom severity, quality of life, and pain mechanisms. As a result, our knowledge about pain locations has shifted our understanding and changed the way we perceive and discuss pain. In the recent two decades, data stemming from simple methods of musculoskeletal pain drawing have helped to reveal the significance of single versus multi-site pain reports. Presently, digital pain mapping studies show that there may be hidden patterns of pain expression within these marked pain sites [5].

\section{Insights revealed}

The number of pain sites marked on a body correlates with disability severity [6], and an increase in the number of sites may serve as a predictor of functional deterioration. An earlier study by Coggon et al. [7] showed that extensive pain, involving six or more pain sites, was more strongly correlated with physical and psychosocial risk factors than that with three or less pain sites. These sites include low back, neck, right/left shoulder, right/left elbow, right/left wrist/hand, and right/left knee. However, co-location of these pain sites rather than their total number emerged as a most peculiar finding. Coggon et al. [7] observed that if additional pain sites were present, they were highly likely to be co-located

Correspondence address: Shellie A. Boudreau, PhD, SMI, Department of Health Science and Technology, Aalborg University, Fredrik Bajers Vej 7, A2-202, Aalborg, Denmark 9220, e-mail: sboudreau@hst.aau.dk

Received: 2018.06.20

Accepted: 2018.09.21

Citation: Boudreau SA. Digital body maps reinvent the way we perceive and discuss pain and discomfort. Physiother Quart. 2018;26(4):1-3; doi: https://doi.org/10.5114/pq.2018.79739 
on the opposite side of the body, particularly for the knee, wrist/hand, and shoulders. In addition to this observation, individuals reporting one pain site were also likely to indicate pain at an adjacent location (on the same side of the body), especially in the case of the upper limb, neck, and shoulder regions.

A study conducted among individuals older than 55 years in the Polish population revealed that the number of pain sites might have a socio-economic impact. Kozak-Szkopek et al. [8] observed that those aged 65 years or older more frequently pointed at pain in low back and lower limbs, and these individuals also reported higher pain intensity and greater use of medical care. The question to be answered is how and why more pain sites occur. A prospective cohort study in the Norwegian population revealed that regardless of the number of pain sites assessed at baseline, symptoms of anxiety and/or depression, sleeping problems, and obesity may account for an increased number of pain sites assessed 11 years later [9]. An additional finding by Mundal et al. [9] was that a higher level of education (e.g. college or
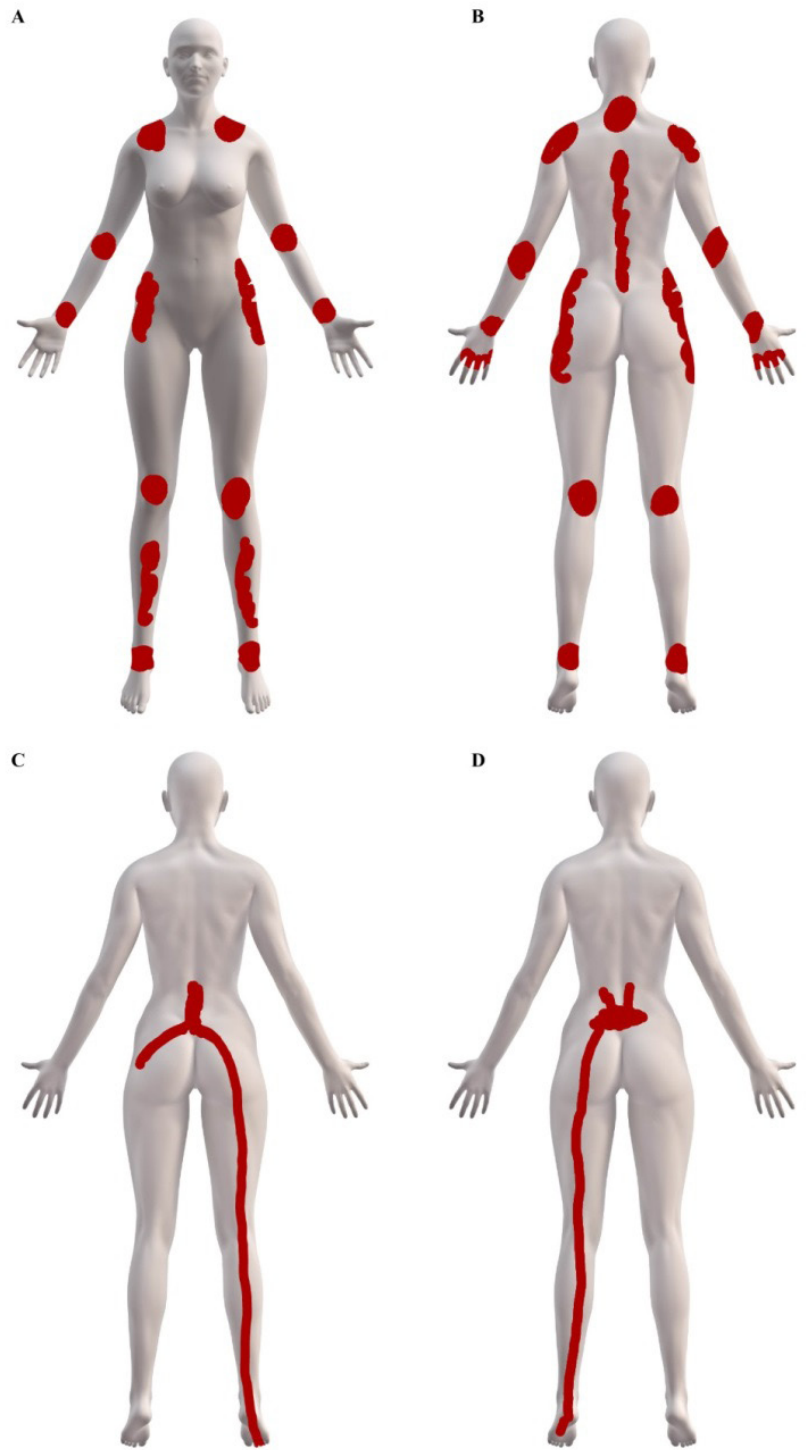

Figure 2. Patient-reported digital pain drawings completed on high-resolution body charts with the use of Navigate Pain, as based on raw data stemming from a reliability study [1].

(A) Front and (B) back pain expressions of an individual diagnosed with fibromyalgia, arthritis, and a herniated disc; (C, D) two separate pain drawings from individuals diagnosed university) was associated with a reduction in the number of pain sites.

The phenomenon of multiple pain sites also overlaps with another concept: that pain may spread beyond the original site of injury. Conceivably, pain that spreads would support the notion of adjacent pain sites emerging as co-location pain sites. However, pain occurring on the opposite side of the body requires a deeper consideration of the mechanisms which may drive the emergence of additional pain sites. In order to establish a complete clinical picture of this phenomenon, we will need more than just a record of multiple pain sites. Instead, technologies capable of capturing the pattern of pain as it unfolds will allow us to discover new pain patterns and reinvent the way we perceive and discuss pain.

\section{Moving forward with digital technologies}

With the introduction of touch-screen technology, patientreported pain drawings can now be acquired with digital applications or drawing platforms as displayed on smartphones and personal computer tablets. These digital applications serve as reliable equivalents to paper-based pain drawings $[1,2,10]$, with added and foreseeable future advantages. In the same way, digital pain drawings provide information about the number of pain sites and pain location. Moreover, the amount of detail, as shown in Figure 2, and easier quantification of pain areas and patterns are more readably achieved with digital solutions. Additional benefits include the ability to contrast one or more pain drawings at different time points, to determine common features within or between patient populations $[4,5]$, and to map other qualities of pain or discomforts [11], such as burning or aching sensations. Indeed, other methods of digitizing pain drawings exist, such as scanning paper drawings and modifying pre-existing paint and drawing tools [4]. Granted, the digitization of pain, by way of pain drawings, has a foreseeable benefit of becoming a digital e-health tool within the clinic and a big data tool for clinical research.

Today, physiotherapists are confronted with increasingly complex patient histories and tighter consultation schedules. Patients with complex histories require considerably more time to communicate their symptoms. Simultaneously, physiotherapists are reliant on the patients' description and accurate recall of their symptoms to understand and guide treatment. Thusly, there is a need for tools to assist clinical workflow, reduce operating expenses, and improve the quality of care. As an example, patient-completed body maps allow the physiotherapist to process information about the location and bodily distribution of pain and discomfort parallelly, rapidly, and automatically. Digital body maps therefore represent an innovative visualization tool that can facilitate communication between the patient and physiotherapist and boost the recognition and understanding of relatively complex health conditions.

Patient-reported pain drawings can be viewed as a digital body map reflecting the area, location, and co-location of multiple pain sites or the spreading of pain. In other words, digital body mapping captures the distribution of bodily pain and discomfort while simultaneously enabling automatic extraction of pain map metrics. It is tempting to speculate how much can be captured with digital body maps and to what extent the recognition and understanding of relatively complex conditions and syndromes are facilitated. Surely, pain drawings acquired daily, weekly, or monthly would help obtain insightful longitudinal data sets and identify symptom trajectories with more ease when reviewed in a clinical setting. Moreover, displaying the pain maps on innovative visualization platforms has a tremendous potential for clinicians 
and researchers. A presentation of longitudinal data sets, for example, stimulates our innate ability to visually process information in parallel about the location and distribution of pain and discomfort rapidly and automatically. At this point in time, researchers and clinicians alike can move beyond locationbased metrics to re-displaying and extracting critical and clinically relevant features about a patient's pain and discomfort as they unfold.

\section{Ethical approval}

The conducted research is not related to either human or animal use.

\section{Acknowledgements}

No funding was provided for this work. Shellie A. Boudreau is supported by the Talent Management Grant (Aalborg University, Denmark).

\section{Disclosure statement}

The author has no financial interest and received no financial benefit from this research.

\section{Conflict of interest}

Shellie A. Boudreau is a co-developer of Navigate Pain v. 1.0 (Android, Aalborg University) and has holdings in Aglance Solutions ApS (Denmark), which licenses a web application of Navigate Pain.

\section{References}

1. Egsgaard LL, Christensen TS, Petersen IM, Brønnum DS, Boudreau SA. Do gender-specific and high-resolution three dimensional body charts facilitate the communication of pain for women? A quantitative and qualitative study. JMIR Hum Factors. 2016;3(2):e19; doi: 10.2196/ humanfactors.5693.

2. Boudreau SA, Badsberg S, Christensen SW, Egsgaard LL. Digital pain drawings: assessing touch-screen technology and 3D body schemas. Clin J Pain. 2016;32(2):139145; doi: 10.1097/AJP.0000000000000230.

3. Cruder C, Falla D, Mangili F, Azzimonti L, Araújo LS, Williamon A, et al. Profiling the location and extent of musicians' pain using digital pain drawings. Pain Pract. 2018; 18(1):53-66; doi: 10.1111/papr.12581.

4. Lluch Girbés E, Dueñas L, Barbero M, Falla D, Baert IA, Meeus $M$, et al. Expanded distribution of pain as a sign of central sensitization in individuals with symptomatic knee osteoarthritis. Phys Ther. 2016;96(8):1196-1207; doi: 10.2522/ptj.20150492.

5. Boudreau SA, Kamavuako EN, Rathleff MS. Distribution and symmetrical patellofemoral pain patterns as revealed by high-resolution 3D body mapping: a cross-sectional study. BMC Musculoskelet Disord. 2017;18(1):160-169; doi: 10.1186/s12891-017-1521-5.

6. Saltychev M, Laimi K. Predicting self-reported disability level by a number of pain sites marked on pain drawing. Int J Rehabil Res. 2018;41(3):276-279; doi: 10.1097/ MRR.0000000000000293.

7. Coggon D, Ntani G, Palmer KT, Felli VE, Harari R, Barrero $\mathrm{LH}$, et al. Patterns of multisite pain and associations with risk factors. Pain. 2013;154(9):1769-1777; doi: 10.1016/j.pain.2013.05.039.

8. Kozak-Szkopek E, Broczek K, Slusarczyk P, Wieczorowska-Tobis K, Klich-Raczka A, Szybalska A, et al. Prevalence of chronic pain in the elderly Polish population - results of the PolSenior study. Arch Med Sci. 2017 ; 13(5):1197-1206; doi: 10.5114/aoms.2015.55270.

9. Mundal I, Bjørngaard JH, Nilsen TI, Nicholl BI, Gråwe RW, Fors EA. Long-term changes in musculoskeletal pain sites in the general population: the HUNT study. J Pain. 2016;17(11):1246-1256; doi: 10.1016/j.jpain.2016.08.006.

10. Matthews M, Rathleff MS, Vicenzino B, Boudreau SA. Capturing patient-reported area of knee pain: a concurrent validity study using digital technology in patients with patellofemoral pain. PeerJ. 2018;6:e4406; doi: 10.7717/ peerj.4406.

11. Neubert TA, Dusch M, Karst M, Beissner F. Designing a tablet-based software app for mapping bodily symptoms: usability evaluation and reproducibility analysis. JMIR Mhealth Uhealth. 2018;6(5):e127; doi: 10.2196/ mhealth.8409. 